\title{
Relations characteristic of theta functions according to quarter periods
}

\author{
Ismet Yildiz, Neslihan Zorlu and Nihan Turan \\ Duzce University, Department of Mathematics, Duzce, Turkey. \\ Received: 4 January 2016, Revised: 5 January 2016, Accepted: 7 January 2016 \\ Published online: 17 January 2016.
}

Abstract: In this study, using the characteristic values $\left[\begin{array}{c}\varepsilon \\ \varepsilon^{\prime}\end{array}\right] \equiv\left[\begin{array}{l}1 \\ 1\end{array}\right],\left[\begin{array}{l}1 \\ 0\end{array}\right],\left[\begin{array}{l}0 \\ 1\end{array}\right],\left[\begin{array}{l}0 \\ 0\end{array}\right](\bmod 2)$ a theorem on the $\frac{1}{2^{r}}$ coefficients of periods of first order theta function according to the $(1, \tau)$ period pair ( for $r \in N^{+}$) is established. The following equalities are also obtained.

$$
\theta\left[\begin{array}{l}
1 \\
1
\end{array}\right]\left(u+\frac{1}{4}\left\{\begin{array}{l}
1 \\
1
\end{array}\right\}, \tau=i \theta\left[\begin{array}{l}
1 \\
0
\end{array}\right]\left(u+\frac{1}{4}\left\{\begin{array}{l}
1 \\
1
\end{array}\right\}, \tau\right)\right.
$$

and

$$
\theta\left[\begin{array}{l}
0 \\
1
\end{array}\right]\left(u+\frac{1}{4}\left\{\begin{array}{l}
1 \\
1
\end{array}\right\}, \tau\right)=\theta\left[\begin{array}{l}
0 \\
0
\end{array}\right]\left(u+\frac{1}{4}\left\{\begin{array}{l}
1 \\
1
\end{array}\right\}, \tau\right)
$$

Keywords: Characteristic values, Theta function, period pair, elliptic function.

\section{Introduction}

Let $\Gamma=S L_{2}(Z)$, we define $\Gamma_{N}$ ( or $\Gamma(N)$ ) for each positive integer $N$ to be subgroup of the modular group $\Gamma$ consisting of those matrices satisfying the condition $\left[\begin{array}{ll}a & b \\ c & d\end{array}\right] \equiv I(\bmod N)$

For unit matrix $I=\left[\begin{array}{ll}1 & 0 \\ 0 & 1\end{array}\right]$ in other words, $a \equiv d \equiv 1(\bmod N)$ and $c \equiv b \equiv 0(\bmod N)[2]$.

We first define a theta characteristic to be a two by one matrix of integers, written $\left[\begin{array}{c}\varepsilon \\ \varepsilon^{\prime}\end{array}\right]$. Next, given a complex number $u$, and another complex number $\tau(\operatorname{Im} \tau)>0, \mathfrak{I}$, to denote the upper half-plane). $Z$ for the set of rational integers and $\Gamma(1)$ for the group. Let $N \geq 1$ be an integer and put

$$
\Gamma_{0}(N)=\left\{\left(\begin{array}{ll}
a & b \\
c & d
\end{array}\right) \in \Gamma(1): c \equiv 0(\bmod N)\right\} .
$$

Let be

$$
\mathrm{U}=\left(\begin{array}{ll}
1 & 1 \\
0 & 1
\end{array}\right), \mathrm{V}=\left(\begin{array}{cc}
0 & -1 \\
1 & 0
\end{array}\right), \mathrm{W}=\left(\begin{array}{ll}
1 & 0 \\
1 & 1
\end{array}\right), \mathrm{P}=\left(\begin{array}{cc}
0 & -1 \\
1 & 1
\end{array}\right)
$$


$\Gamma_{U}(2), \Gamma_{V}(2)$ and $\Gamma_{W}(2)$ are defined by

$$
\begin{array}{lll}
\Gamma_{U}(2)=\{S \in \Gamma(1): & S \equiv I \text { or } & S \equiv U(\operatorname{Mod} 2)\} \\
\Gamma_{V}(2)=\{S \in \Gamma(1): & S \equiv I \text { or } & S \equiv V(\operatorname{Mod} 2)\} \\
\Gamma_{W}(2)=\{S \in \Gamma(1): & S \equiv I \text { or } & S \equiv W(\operatorname{Mod} 2)\}
\end{array}
$$

were $I$ is the unit matrix. The three subgroups $\Gamma_{U}(2), \Gamma_{V}(2)$ and $\Gamma_{W}(2)$ are conjugate. The subgroup $\theta$ of $\Gamma(1)$ is generated by $\mathrm{U}$ and $\mathrm{V}$. For an odd positive integer $n$, the set of elements in $\theta$ of the from

$$
\left(\begin{array}{ll}
a & b \\
n c & d
\end{array}\right)
$$

is a subgroup of which will be denoted $\theta(n)[3]$.

Definition 1. For $u \in C, \tau \in \mathfrak{I}$ and characteristic value $\left[\begin{array}{c}\varepsilon \\ \varepsilon^{\prime}\end{array}\right]$, the function defined as

$$
\left[\begin{array}{c}
\varepsilon \\
\varepsilon^{\prime}
\end{array}\right](u, \tau)=\sum_{n=-\infty}^{\infty} \exp \left\{\left(n+\frac{\varepsilon}{2}\right)^{2} \pi i \tau+2 \pi i\left(n+\frac{\varepsilon}{2}\right)\left(u+\frac{\varepsilon^{\prime}}{2}\right)\right\}
$$

is called first order theta function [1].

Definition 2. A half-period is half of a period ( in particular a complex vector), written

$$
\left[\begin{array}{c}
\mu \\
\mu^{\prime}
\end{array}\right] \equiv \frac{1}{2}\left\{\begin{array}{c}
\mu \\
\mu^{\prime}
\end{array}\right\}=\frac{\mu^{\prime}}{2}+\frac{\mu \tau}{2} .
$$

A reduced half-period is half period in which $\mu$ and $\mu^{\prime}$ equal 0 or 1 where $\mu$ and $\mu^{\prime}$ are integers [1].

In the present paper, whenever the integers $\mu$ and $\mu^{\prime}$ will be as $\mu=1$ and $\mu^{\prime}=1$, unless otherwise stated. In this study,

$$
\left[\begin{array}{c}
\varepsilon \\
\varepsilon^{\prime}
\end{array}\right] \equiv\left[\begin{array}{l}
1 \\
1
\end{array}\right],\left[\begin{array}{l}
1 \\
0
\end{array}\right],\left[\begin{array}{l}
0 \\
1
\end{array}\right],\left[\begin{array}{l}
0 \\
0
\end{array}\right](\bmod 2)
$$

values of characteristic are used. When the periodicity of the function $\theta\left[\begin{array}{c}\varepsilon \\ \varepsilon^{\prime}\end{array}\right](u, \tau)$ for $(1, \tau)$ period pair is examined.

$$
\begin{aligned}
{\left[\begin{array}{c}
\varepsilon \\
\varepsilon^{\prime}
\end{array}\right](u+1, \tau) } & =\sum_{n=-\infty}^{\infty} \exp \left\{\left(n+\frac{\varepsilon}{2}\right)^{2} \pi i \tau+2 \pi i\left(n+\frac{\varepsilon}{2}\right)\left(u+1+\frac{\varepsilon^{\prime}}{2}\right)\right\} \\
& =\sum_{n=-\infty}^{\infty} \exp \left\{\left(n+\frac{\varepsilon}{2}\right)^{2} \pi i \tau+2 \pi i\left(n+\frac{\varepsilon}{2}\right)\left(u+\frac{\varepsilon^{\prime}}{2}\right)+n 2 \pi i+\pi i \varepsilon\right\} \\
& =\left((-1)^{\varepsilon \cdot \theta}\left[\begin{array}{c}
\varepsilon \\
\varepsilon^{\prime}
\end{array}\right](u, \tau)\right),
\end{aligned}
$$


also

$$
\begin{aligned}
{\left[\begin{array}{c}
\varepsilon \\
\varepsilon^{\prime}
\end{array}\right](u+\tau, \tau) } & =\sum_{n=-\infty}^{\infty} \exp \left\{\left(n+\frac{\varepsilon}{2}\right)^{2} \pi i \tau+2 \pi i\left(n+\frac{\varepsilon}{2}\right)\left(u+\tau+\frac{\varepsilon^{\prime}}{2}\right)\right\} \\
& =\sum_{n=-\infty}^{\infty} \exp \left\{\left(n+\frac{\varepsilon}{2}\right)^{2} \pi i \tau+2 \pi i\left(n+\frac{\varepsilon}{2}\right)\left(u+\frac{\varepsilon^{\prime}}{2}\right)+n 2 \pi i \tau+\pi i \tau \varepsilon\right\} \\
& =(-1)^{\varepsilon^{\prime}} \exp (-\pi i \tau-2 \pi i u) \theta\left[\begin{array}{c}
\varepsilon \\
\varepsilon^{\prime}
\end{array}\right](u, \tau) .
\end{aligned}
$$

and

$$
\begin{aligned}
{\left[\begin{array}{c}
\varepsilon \\
\varepsilon^{\prime}
\end{array}\right](u+\tau+1, \tau) } & =\sum_{n=-\infty}^{\infty} \exp \left\{\left(n+\frac{\varepsilon}{2}\right)^{2} \pi i \tau+2 \pi i\left(n+\frac{\varepsilon}{2}\right)\left(u+\tau+1+\frac{\varepsilon^{\prime}}{2}\right)\right\} \\
& =\sum_{n=-\infty}^{\infty} \exp \left\{\left(n+\frac{\varepsilon}{2}\right)^{2} \pi i \tau+2 \pi i\left(n+\frac{\varepsilon}{2}\right)\left(u+\frac{\varepsilon^{\prime}}{2}\right)+n 2 \pi i \tau+\pi i \tau \varepsilon\right\} \\
& =(-1)^{\varepsilon} \exp \left(-\pi i \tau-2 \pi i u-\pi i \varepsilon^{\prime}\right) \theta\left[\begin{array}{c}
\varepsilon \\
\varepsilon^{\prime}
\end{array}\right](u, \tau) .
\end{aligned}
$$

By using $\eta_{1}=(-1)^{\varepsilon}, \eta_{2}=(-1)^{\varepsilon^{\prime}} \exp (-\pi i \tau-2 \pi i u)$ and $\eta_{3}=(-1)^{\varepsilon} \exp \left(-\pi i \tau-2 \pi i u-\pi i \varepsilon^{\prime}\right)$ we obtain

$$
\left[\begin{array}{c}
\varepsilon \\
\varepsilon^{\prime}
\end{array}\right](u+\tau+1, \tau)=\eta_{3} \cdot \theta\left[\begin{array}{c}
\varepsilon \\
\varepsilon^{\prime}
\end{array}\right](u, \tau) .
$$

As it is seen here, for $\eta_{3}=1$, because $\theta\left[\begin{array}{c}\varepsilon \\ \varepsilon^{\prime}\end{array}\right](u, \tau)$ is doubly periodic, it would be an elliptic function.

\section{Theorem 1.}

$$
\theta\left[\begin{array}{c}
\varepsilon \\
\varepsilon^{\prime}
\end{array}\right]\left(u+\frac{1}{2^{r}}+\frac{\tau}{2^{r}}, \tau\right)=\exp \left\{-\frac{1}{4^{r}}(\tau+2) \pi i-\frac{1}{2^{r}}\left(2 u+\varepsilon^{\prime}\right) \pi i\right\} \cdot \theta\left[\begin{array}{c}
\varepsilon+\frac{1}{2^{r-1}} \\
\varepsilon^{\prime}+\frac{1}{2^{r-1}}
\end{array}\right](u, \tau)
$$

where $r \in N^{+}$.

Proof.

$$
\begin{aligned}
\theta\left[\begin{array}{c}
\varepsilon \\
\varepsilon^{\prime}
\end{array}\right]\left(u+\frac{1}{2^{r}}+\frac{\tau}{2^{r}}, \tau\right) & =\sum_{n=-\infty}^{\infty} \exp \left\{\left(n+\frac{\varepsilon}{2}\right)^{2} \pi i \tau+2 \pi i\left(n+\frac{\varepsilon}{2}\right)\left(u+\frac{1}{2^{r}}+\frac{\tau}{2^{r}}++\frac{\varepsilon^{\prime}}{2}\right)\right\} \\
& =\sum_{n=-\infty}^{\infty} \exp \left\{\left(n+\frac{\varepsilon}{2}\right)^{2} \pi i \tau+2 \pi i\left(n+\frac{\varepsilon}{2}\right)\left(u+\frac{\varepsilon^{\prime}}{2}\right)+\frac{n \pi i \tau}{2^{r-1}}+\frac{\pi i \tau}{2^{r-1}}+\frac{\pi i \tau \varepsilon^{\prime}}{2^{r}}+\frac{\pi i \varepsilon^{\prime}}{2^{r}}\right\} .
\end{aligned}
$$

On the other hand, the reduced representative of an arbitrary characteristic

$$
\left[\begin{array}{c}
\varepsilon \\
\varepsilon^{\prime}
\end{array}\right]
$$

to be that reduced characteristic whose entries are the least nonnegative residues $(\bmod 2)$ of $\varepsilon$ and $\varepsilon^{\prime}$.

There are four reduced characteristics $\left[\begin{array}{l}0 \\ 0\end{array}\right],\left[\begin{array}{l}0 \\ 1\end{array}\right],\left[\begin{array}{l}1 \\ 0\end{array}\right],\left[\begin{array}{l}1 \\ 1\end{array}\right]$. But $\theta\left[\begin{array}{l}1 \\ 1\end{array}\right](0, \tau) \equiv 0$. 
$\theta\left[\begin{array}{c}\varepsilon+\frac{1}{2^{r-1}} \\ \varepsilon^{\prime}+\frac{1}{2^{r-1}}\end{array}\right](u, \tau)=\sum_{n=-\infty}^{\infty} \exp \left\{\left(n+\frac{\varepsilon}{2}\right)^{2} \pi i \tau+2 \pi i\left(n+\frac{\varepsilon}{2}\right)\left(u+\frac{\varepsilon^{\prime}}{2}\right)+\frac{n \pi i \tau}{2^{r-1}}+\frac{2 \pi i u}{2^{r}}+\frac{2 \pi i \varepsilon^{\prime}}{2^{r}}+\frac{\pi i \tau \varepsilon}{2^{r}}+\frac{n \pi i}{2^{r-1}}+\frac{\pi i \tau}{4^{r}}+\frac{2 \pi i}{4^{r}}+\frac{\pi i \varepsilon}{2^{r}}\right\}$ and

$$
\begin{aligned}
& \exp \left\{-\frac{1}{4^{r}}(\tau+2) \pi i-\frac{1}{2^{r}}\left(2 u+\varepsilon^{\prime}\right) \pi i\right\} \theta\left[\begin{array}{c}
\varepsilon+\frac{1}{2^{r-1}} \\
\varepsilon^{\prime}+\frac{1}{2^{r-1}}
\end{array}\right](u, \tau) \\
& =\sum_{n=-\infty}^{\infty} \exp \left\{\left(n+\frac{\varepsilon}{2}\right)^{2} \pi i \tau+2 \pi i\left(n+\frac{\varepsilon}{2}\right)\left(u+\frac{\varepsilon^{\prime}}{2}\right)+\frac{n \pi i \tau}{2^{r-1}}+\frac{\pi i \tau \varepsilon}{2^{r}}+\frac{n \pi i}{2^{r-1}}+\frac{\pi i \varepsilon}{2^{r}}\right\}
\end{aligned}
$$

By the theorem given above we can obtain the following characteristic equalities for $u=0$ value of the complex variable

(a)

$$
\begin{aligned}
& \theta\left[\begin{array}{l}
1 \\
1
\end{array}\right]\left(0+\frac{1}{2^{r}}+\frac{\tau}{2^{r}}, \tau\right)=\exp \left\{-\frac{1}{4^{r}}(\tau+2) \pi i-\frac{1}{2^{r}} \pi i\right\} \cdot \theta\left[\begin{array}{c}
1+\frac{1}{2^{r-1}} \\
1+\frac{1}{2^{r-1}}
\end{array}\right](0, \tau) \\
& =\sum_{n=-\infty}^{\infty} \exp \left\{\left(n+\frac{1}{2}+\frac{1}{2^{r}}\right)^{2} \pi i \tau+2 \pi i\left(n+\frac{1}{2}+\frac{1}{2^{r}}\right)\left(0+\frac{1}{2}+\frac{1}{2^{r}}\right)-\frac{\pi i \tau}{4^{r}}+\frac{\pi i}{2^{r-1}}+\frac{\pi i}{2^{r}}\right\} \\
& =\sum_{n=-\infty}^{\infty} \exp \left\{\left(n+\frac{1}{2}\right)^{2} \pi i \tau+\frac{n \pi i \tau}{2^{r-1}}+\frac{\pi i \tau}{2^{r}}+\frac{n \pi i}{2^{r-1}}+\frac{\pi i}{2^{r}}+\frac{\pi i}{2}+n \pi i\right\}
\end{aligned}
$$

and

$$
\begin{aligned}
& \theta\left[\begin{array}{l}
1 \\
0
\end{array}\right]\left(0+\frac{1}{2^{r}}+\frac{\tau}{2^{r}}, \tau\right)=\exp \left\{-\frac{1}{4^{r}}(\tau+2) \pi i\right\} \cdot \theta\left[\begin{array}{c}
1+\frac{1}{2^{r-1}} \\
0+\frac{1}{2^{r-1}}
\end{array}\right](0, \tau) \\
& =\sum_{n=-\infty}^{\infty} \exp \left\{\left(n+\frac{1}{2}+\frac{1}{2^{r}}\right)^{2} \pi i \tau+2 \pi i\left(n+\frac{1}{2}+\frac{1}{2^{r}}\right)\left(0+\frac{1}{2}+\frac{1}{2^{r}}\right)-\frac{\pi i \tau}{4^{r}}-\frac{\pi i}{2^{r-1}}\right\} \\
& =\sum_{n=-\infty}^{\infty} \exp \left\{\left(n+\frac{1}{2}\right)^{2} \pi i \tau+\frac{n \pi i \tau}{2^{r-1}}+\frac{\pi i \tau}{2^{r}}+\frac{n \pi i}{2^{r-1}}+\frac{\pi i}{2^{r}}+\frac{\pi i}{2}+n \pi i\right\} .
\end{aligned}
$$

From the equations (4) and (5), we can get the following equality

$$
\exp \left\{-\frac{1}{4^{r}}(\tau+2) \pi i-\frac{1}{2^{r}} \pi i\right\} \theta\left[\begin{array}{c}
1+\frac{1}{2^{r-1}} \\
1+\frac{1}{2^{r-1}}
\end{array}\right](0, \tau)=\exp \left\{-\frac{1}{4^{r}}(\tau+2) \pi i\right\} \theta\left[\begin{array}{c}
1+\frac{1}{2^{r-1}} \\
0+\frac{1}{2^{r-1}}
\end{array}\right](0, \tau) .
$$

(b)

$$
\begin{aligned}
& \theta\left[\begin{array}{l}
0 \\
0
\end{array}\right]\left(0+\frac{1}{2^{r}}+\frac{\tau}{2^{r}}, \tau\right)=\exp \left\{-\frac{1}{4^{r}}(\tau+2) \pi i\right\} \cdot \theta\left[\begin{array}{c}
0+\frac{1}{2^{r-1}} \\
0+\frac{1}{2^{r-1}}
\end{array}\right](0, \tau) \\
& =\sum_{n=-\infty}^{\infty} \exp \left\{\left(n+\frac{1}{2^{r}}\right)^{2} \pi i \tau+2 \pi i\left(n+\frac{1}{2^{r}}\right)\left(0+\frac{1}{2^{r}}\right)-\frac{\pi i \tau}{4^{r}}+\frac{\pi i}{2^{r-1}}\right\} \\
& =\sum_{n=-\infty}^{\infty} \exp \left\{n^{2} \pi i \tau+\frac{n \pi i \tau}{2^{r-1}}+\frac{n \pi i}{2^{r-1}}\right\}
\end{aligned}
$$


and

$$
\begin{aligned}
& \theta\left[\begin{array}{l}
0 \\
1
\end{array}\right]\left(0+\frac{1}{2^{r}}+\frac{\tau}{2^{r}}, \tau\right)=\exp \left\{-\frac{1}{4^{r}}(\tau+2) \pi i-\frac{\pi i}{2^{r}}\right\} \cdot \theta\left[\begin{array}{l}
0+\frac{1}{2^{r-1}} \\
1+\frac{1}{2^{r-1}}
\end{array}\right](0, \tau) \\
& =\sum_{n=-\infty}^{\infty} \exp \left\{\left(n+\frac{1}{2^{r}}\right)^{2} \pi i \tau+2 \pi i\left(n+\frac{1}{2^{r}}\right)\left(0+\frac{1}{2}+\frac{1}{2^{r}}\right)-\frac{\pi i \tau}{4^{r}}-\frac{\pi i}{2^{r-1}}-\frac{\pi i}{2^{r}}\right\} \\
& =\sum_{n=-\infty}^{\infty} \exp \left\{n^{2} \pi i \tau+\frac{n \pi i \tau}{2^{r-1}}+n \pi i+\frac{n \pi i}{2^{r-1}}\right\}
\end{aligned}
$$

If $n=2 k \in Z$, then from the equalities (6) and (7) the following is obtained

$$
\exp \left\{-\frac{1}{4^{r}}(\tau+2) \pi i-\frac{1}{2^{r}} \pi i\right\} \theta\left[\begin{array}{c}
0+\frac{1}{2^{r-1}} \\
1+\frac{1}{2^{r-1}}
\end{array}\right](0, \tau)=\exp \left\{-\frac{1}{4^{r}}(\tau+2) \pi i\right\} \theta\left[\begin{array}{c}
0+\frac{1}{2^{r-1}} \\
0+\frac{1}{2^{r-1}}
\end{array}\right](0, \tau)
$$

With the help of this theorem proved, transformations among theta functions can be found for characteristic value $\left[\begin{array}{c}\varepsilon \\ \varepsilon^{\prime}\end{array}\right]$ according to all multiples $\frac{1}{2^{r}}$ of the periods. The subject that should be discussed here is; characteristic values

$$
\left[\begin{array}{c}
\varepsilon \\
\varepsilon^{\prime}
\end{array}\right] \equiv\left[\begin{array}{l}
1 \\
1
\end{array}\right],\left[\begin{array}{l}
1 \\
0
\end{array}\right],\left[\begin{array}{l}
0 \\
1
\end{array}\right],\left[\begin{array}{l}
0 \\
0
\end{array}\right](\bmod 2)
$$

of first order theta function can be expressed as characteristic values

$$
\left[\begin{array}{c}
\varepsilon+\frac{1}{2^{r-1}} \\
\varepsilon^{\prime}+\frac{1}{2^{r-1}}
\end{array}\right]
$$

This situation has proved that theta functions are generalized as characteristic values

$$
\left[\begin{array}{c}
\varepsilon+\frac{1}{2^{r-1}} \\
\varepsilon^{\prime}+\frac{1}{2^{r-1}}
\end{array}\right]
$$

With the help of this alternative formula above, we can get the following equalities according to quarter-periods. If $\left[\begin{array}{c}\varepsilon \\ \varepsilon^{\prime}\end{array}\right] \equiv\left[\begin{array}{l}1 \\ 1\end{array}\right](\bmod 2)$ then

$$
\begin{aligned}
& \theta\left[\begin{array}{l}
1 \\
1
\end{array}\right]\left(u+\frac{1}{4}\left\{\begin{array}{l}
1 \\
1
\end{array}\right\}, \tau\right)=\sum_{n} \exp \left\{\left(n+\frac{1}{2}\right)^{2} \pi i \tau+2 \pi i\left(n+\frac{1}{2}\right)\left(u+\frac{1}{4}\left\{\begin{array}{l}
1 \\
1
\end{array}\right\}+\frac{1}{2}\right\}\right. \\
& =i e^{-\frac{\pi i \tau}{4}} \sum_{n}(-1)^{n} \exp \left\{\left(n+\frac{1}{2}\right)^{2} \pi i \tau+(2 n+1) \pi i u+\frac{n \pi i}{2}+\frac{n \pi i \tau}{2}+\frac{\pi i \tau}{4}+\frac{\pi i}{4}\right\} .
\end{aligned}
$$

If $\left[\begin{array}{c}\varepsilon \\ \varepsilon^{\prime}\end{array}\right] \equiv\left[\begin{array}{l}1 \\ 0\end{array}\right](\bmod 2)$ then

$$
\begin{aligned}
& \theta\left[\begin{array}{l}
1 \\
0
\end{array}\right]\left(u+\frac{1}{4}\left\{\begin{array}{l}
1 \\
1
\end{array}\right\}, \tau\right)=\sum_{n} \exp \left\{\left(n+\frac{\varepsilon}{2}\right)^{2} \pi i \tau+2 \pi i\left(n+\frac{1}{2}\right)\left(u+\frac{1}{4}\left\{\begin{array}{l}
1 \\
1
\end{array}\right\}\right\}\right. \\
& =e^{-\frac{\pi i \tau}{4}} \sum_{n} \exp \left\{\left(n+\frac{1}{2}\right)^{2} \pi i \tau+(2 n+1) \pi i u+\frac{n \pi i}{2}+\frac{n \pi i \tau}{2}+\frac{\pi i \tau}{4}+\frac{\pi i}{4}\right\} \text {. }
\end{aligned}
$$


Using the equations (8) and (9) we can get

$$
\frac{\theta\left[\begin{array}{l}
1 \\
1
\end{array}\right]\left(u+\frac{1}{4}\left\{\begin{array}{l}
1 \\
1
\end{array}\right\}, \tau\right)}{\theta\left[\begin{array}{l}
1 \\
0
\end{array}\right]\left(u+\frac{1}{4}\left\{\begin{array}{l}
1 \\
1
\end{array}\right\}, \tau\right)}=\frac{i e^{-\frac{\pi i \tau}{4}} \sum_{n}(-1)^{n} \exp \left\{\left(n+\frac{1}{2}\right)^{2} \pi i \tau+(2 n+1) \pi i u+\frac{n \pi i}{2}+\frac{n \pi i \tau}{2}+\frac{\pi i \tau}{4}+\frac{\pi i}{4}\right\}}{e^{-\frac{\pi i \tau}{4}} \sum_{n} \exp \left\{\left(n+\frac{1}{2}\right)^{2} \pi i \tau+(2 n+1) \pi i u+\frac{n \pi i}{2}+\frac{n \pi i \tau}{2}+\frac{\pi i \tau}{4}+\frac{\pi i}{4}\right\}} .
$$

(i) If $n$ is 0 or even integer then,

$$
\theta\left[\begin{array}{l}
1 \\
1
\end{array}\right]\left(u+\frac{1}{4}\left\{\begin{array}{l}
1 \\
1
\end{array}\right\}, \tau\right)=i \theta\left[\begin{array}{l}
1 \\
0
\end{array}\right]\left(u+\frac{1}{4}\left\{\begin{array}{l}
1 \\
1
\end{array}\right\}, \tau\right)
$$

(ii) If $n$ is odd integer then

$$
\theta\left[\begin{array}{l}
1 \\
1
\end{array}\right]\left(u+\frac{1}{4}\left\{\begin{array}{l}
1 \\
1
\end{array}\right\}, \tau\right)=-i \theta\left[\begin{array}{l}
1 \\
0
\end{array}\right]\left(u+\frac{1}{4}\left\{\begin{array}{l}
1 \\
1
\end{array}\right\}, \tau\right)
$$

If $\left[\begin{array}{c}\varepsilon \\ \varepsilon^{\prime}\end{array}\right] \equiv\left[\begin{array}{l}0 \\ 1\end{array}\right](\bmod 2)$ then

$$
\begin{gathered}
\theta\left[\begin{array}{l}
0 \\
1
\end{array}\right]\left(u+\frac{1}{4}\left\{\begin{array}{l}
1 \\
1
\end{array}\right\}, \tau\right)=\sum_{n} \exp \left\{n^{2} \pi i \tau+2 n \pi i\left(u+\frac{1}{4}\left\{\begin{array}{l}
1 \\
1
\end{array}\right\}+\frac{1}{2}\right\}\right. \\
=\sum_{n}(-1)^{n} \exp \left\{n^{2} \pi i \tau+2 n \pi i u+\frac{n \pi i}{2}+\frac{n \pi i \tau}{2}\right\} .
\end{gathered}
$$

If $\left[\begin{array}{c}\varepsilon \\ \varepsilon^{\prime}\end{array}\right] \equiv\left[\begin{array}{l}0 \\ 0\end{array}\right](\bmod 2)$ then

$$
\begin{gathered}
\theta\left[\begin{array}{l}
0 \\
0
\end{array}\right]\left(u+\frac{1}{4}\left\{\begin{array}{l}
1 \\
1
\end{array}\right\}, \tau\right)=\sum_{n} \exp \left\{n^{2} \pi i \tau+2 n \pi i\left(u+\frac{1}{4}\left\{\begin{array}{l}
1 \\
1
\end{array}\right\}\right\}\right. \\
=\sum_{n} \exp \left\{n^{2} \pi i \tau+2 n \pi i u+\frac{n \pi i}{2}+\frac{n \pi i \tau}{2}\right\}
\end{gathered}
$$

From the equations (10) and (11) we obtain

$$
\frac{\theta\left[\begin{array}{l}
0 \\
1
\end{array}\right]\left(u+\frac{1}{4}\left\{\begin{array}{l}
1 \\
1
\end{array}\right\}, \tau\right)}{\theta\left[\begin{array}{l}
0 \\
0
\end{array}\right]\left(u+\frac{1}{4}\left\{\begin{array}{l}
1 \\
1
\end{array}\right\}, \tau\right)}=\frac{\sum_{n}(-1)^{n} \exp \left\{n^{2} \pi i \tau+2 n \pi i u+\frac{n \pi i}{2}+\frac{n \pi i \tau}{2}\right\}}{\sum_{n} \exp \left\{n^{2} \pi i \tau\left(2 n \pi i u+\frac{n \pi i}{2}+\frac{n \pi i \tau}{2}\right\}\right.} .
$$

(iii) If $n$ is 0 or even integer then

$$
\theta\left[\begin{array}{l}
0 \\
1
\end{array}\right]\left(u+\frac{1}{4}\left\{\begin{array}{l}
1 \\
1
\end{array}\right\}, \tau\right)=\theta\left[\begin{array}{l}
0 \\
0
\end{array}\right]\left(u+\frac{1}{4}\left\{\begin{array}{l}
1 \\
1
\end{array}\right\}, \tau\right)
$$

(iv) If $n$ is odd integer then

$$
\theta\left[\begin{array}{l}
0 \\
1
\end{array}\right]\left(u+\frac{1}{4}\left\{\begin{array}{l}
1 \\
1
\end{array}\right\}, \tau\right)=-\theta\left[\begin{array}{l}
0 \\
0
\end{array}\right]\left(u+\frac{1}{4}\left\{\begin{array}{l}
1 \\
1
\end{array}\right\}, \tau\right)
$$




\section{Conclusion}

A general relation has been obtained by using $(1, r)$ periodic couples of first order theta functions according to the multiples of $1 / 2^{r}$ of these couples for $r \in N$. Furthermore an example has been given by using these relation for $r=2$ according to the multiples of $1 / 4$.

\section{References}

[1] Gregory L. Wilson, A family of modular functions Arising from the theta function London Math. Soc. (3),55,1987, London.

[2] Harry E. Rauch., Elliptic functions, theta functions and Riemann surfaces. The Williams and Wilkins Company Baltimore. 7579.(1973). Maryland.

[3] S. Raghavan., Cups Forms of Degre 2 and weight. Jour. of. Math. Ann.(224), 14156.(1976).

[4] Yıldı İsmet., On extension of modular transformations over the modular group by reflection. Journal of Applied Mathematics and Computation. Volume 153,Issue 1, p.111-116. (2004).

[5] Zhi-Guo Liu1. An addition formula for the Jacobian theta function and its applications. Advances in Mathematics Volume 212, Issue 1, 20 June 2007, Pages 389-40.

[6] István Mező. Several special values of Jacobi theta functions. (Submitted on 14 Jun 2011 (v1), last revised 24 Sep 2013 (this version, v3)). Cornell Universty Library.

[7] Noam D. Elkies, Lattices, Linear Codes and Invariants. Notices of the AMS, Volume 47, Number 10 (November 2000).

[8] Don Zagier. Ramanujan's Mock Theta Functions And Their Applications. Seminaire Bourbaki Novembre 2007 .60'eme ann'ee, 2006-2007, no 986.

[9] Theta Functions. Irish Math. Soc. Bulletin 60 (2007), 91-112. 